\title{
Anabases
}

ANABASES Traditions et réceptions de l'Antiquité

\section{Occultations de Zalmoxis et occultation de l'histoire. Un aspect du dossier Mircea Eliade}

Dan Dana

\section{(2) OpenEdition}

1 Journals

Édition électronique

URL : http://journals.openedition.org/anabases/3042

DOI : 10.4000/anabases.3042

ISSN : 2256-9421

Éditeur

E.R.A.S.M.E.

Édition imprimée

Date de publication : 1 mars 2007

Pagination : 11-25

ISSN : 1774-4296

\section{Référence électronique}

Dan Dana, « Occultations de Zalmoxis et occultation de l'histoire. Un aspect du dossier Mircea Eliade

», Anabases [En ligne], 5 | 2007, mis en ligne le 01 janvier 2012, consulté le 21 octobre 2019. URL

http://journals.openedition.org/anabases/3042 ; DOI : 10.4000/anabases.3042

(c) Anabases 


\section{Occultations de Zalmoxis et occultation de l'histoire. Un aspect du dossier Mircea Eliade}

DAN DANA

$\mathrm{C}$ ETTE ÉTUdE SE PROPOSE d'analyser l'interprétation de Mircea Eliade sur un sujet particulier - car il touchait au passé de sa patrie -, à savoir son analyse de Zalmoxis (1970). Mon intention n'est pas d'aborder le dossier sur le silence d'Eliade, très actuel en Occident mais dérangeant en Roumanie ; en revanche, je voudrais insister sur le lien entre sa vie, ses convictions idéologiques et son œuvre : matière à réfléchir sur la mythologie personnelle, qu'on rencontre dans tous ses écrits ${ }^{1}$.

\section{Qui est Zalmoxis?}

Pour Zalmoxis, dieu des Gètes, le texte d'Hérodote (IV, 94-96) constitue le point de départ d'une tradition littéraire multiforme. Il n'y a aucune source archéologique, iconographique ou épigraphique à son sujet; seule la dimension littéraire reste à analyser, fruit d'innombrables interprétations successives. Esclave et disciple barbare de Pythagore, civilisateur des Gètes, Zalmoxis reste un exemple de "sagesse barbare " et une des figures de l'altérité par lesquelles les intellectuels grecs pensaient leur propre culture $^{2}$. Mais c'est également un moyen privilégié d'analyser le regard et le jugement portés par toute une science occidentale sur un peuple barbare et marginal, et par l'in-

1 Je développe ici une partie de ma thèse Enjeux et controverses autour du mythe de Zalmoxis (EHESS, 2005). Voir maintenant M. ELIADE, Jurnalul portughez şi alte scrieri [Le journal portugais et d'autres écrits], I-II, Bucarest, 2006. On y retrouve son extrême mégalomanie, la gestation de ses futurs livres, et son engagement dans le mouvement d'extrême droite roumain.

2 F. Hartog, Le miroir d'Hérodote. Essai sur la représentation de l'autre, Paris, 200133, p. 164198 [= 1980] ("Salmoxis : le Pythagore des Gètes"). 
telligentsia roumaine sur son passé, sur cette catégorie des " ancêtres » dans laquelle on aime se projeter et se reconnaître.

Tous les paradigmes de l'histoire des religions sont largement reflétés dans l'interprétation du dieu gète : naturalisme, totémisme, chamanisme, religion initiatique, religion trifonctionnelle. Au XIXe siècle, l'historiographie roumaine s'approprie Zalmoxis : il symbolise désormais la haute spiritualité des ancêtres autochtones, d'une Dacie censée préfigurer l'État national roumain. Cette idéologie du "spécifiquement national » se prêtait aussi à une exploitation politique, aussi bien par l'extrême droite dans les années 1930 que pendant le national-communisme de Ceauşescu, où l'on assiste à un développement fantastique des théories autochtonistes (la thracomanie). Le complexe d'infériorité par rapport à l'Occident habitant durablement l'identité roumaine, comme toute "culture mineure ", le renversement était d'autant plus séduisant : les ancêtres daces avaient une religion spirituelle et supérieure aux autres, préfigurant le monothéisme et le christianisme constitutifs de l'Occident. Lire les exégèses livrées au sujet de la "spiritualité dace » jette une lumière nouvelle sur les diverses modalités de réécrire le passé roumain, de reconfigurer les contours d'une identité incertaine, entre ancien et nouveau, entre Occident et Orient, entre négation de soi ou de l'histoire et affirmation d'une excellence anhistorique. La thèse d'Eliade doit être considérée dans ce tissu délicat de présupposés et d’usages, jamais innocents.

\section{Le Zalmoxis d'Eliade : dieu de l'initiation et des mystères (1970)}

Le paradigme interprétatif actuel propose de voir dans le culte de Zalmoxis une religion initiatique. L'arrière-plan est fourni par les rapprochements opérés dès le XIXe siècle entre le culte dionysiaque et Zalmoxis, tradition illustrée par E. Rohde (Psychè). En Roumanie, dans les années 1930 déjà, Jean Coman, ami et collaborateur d'Eliade à la revue Zalmoxis, propose une vision extrêmement spiritualisée du dieu gète, prêchant le monothéisme et l'immortalité de l'âme ${ }^{3}$.

La thèse d'une religion gète fondée sur des mystères ne sera affirmée de manière «définitive » que par Mircea Eliade (1907-1986) ${ }^{4}$. La religion dace n'est pas centrale

3 J. COMAN, “Zalmoxis. Un grand problème gète ”, Zalmoxis 2 (1939), p. 79-110 ; ID., "L'immortalité chez les Thraco-Géto-Daces ”, Revue de l'Histoire des Religions 198 (1981), p. $243-278$.

4

N. SPINETO, "Le comparatisme de Mircea Eliade”, in F. BeEspflug-F. Dunand (éd.), Le comparatisme en histoire des religions. Actes du Colloque International de Strasbourg (18-20 septembre 1996), Paris, 1997, p. 93-108 ; ID., " Mircea Eliade : éléments pour un bilan historiographique ”, in J. RIES-N. SPINETO (éd.), Deux explorateurs de la pensée humaine. Georges Dumézil et Mircea Eliade, Turnhout, 2003, p. 137-182 ; Ph. BORGEAUD, Exercices de mythologie, Genève, 2004, p. 179-205 ; M. OlEnder, La chasse aux évidences. Sur quelques formes de racisme entre mythe et histoire. 1978-2005, Paris, 2005, p. 147-153. Le 
dans son œuvre monumentale d'intention universelle ; plutôt que l'antiquité, Eliade privilégie le folklore, vu comme témoin privilégié d'un état spirituel pré- et protohistorique, héritier d'un substrat archaïque. Cet intérêt pour le folklore et l'" essence » de la spiritualité roumaine s'accroît dans la deuxième partie des années trente - les années même de son engagement politique dans la Garde de Fer. L'histoire des religions et l'intérêt pour le folklore sont réunis dans la revue Zalmoxis (I-III, Paris, 1938-1942), nom heureusement choisi selon Pettazzoni ${ }^{5}$, revue qui sera de grand secours à Eliade après la guerre. Parmi les sources roumaines, il faut noter son admiration pour L. Blaga, qui avait réévalué l'importance du fonds autochtone gète 6 .

Établi après la guerre à Paris, où il est appuyé par Dumézil, Eliade devient vite connu et apprécié, ce qui lui permet, à la fin des années 1950, de relancer sa carrière à l'université de Chicago ; aux États-Unis, il devient l'historien des religions le plus connu. Certes, partout dans ses écrits, Eliade est intéressé par l'universel et animé du désir de dépasser le "provincialisme "; quant aux sujets roumains, ils sont toujours analysés dans un cadre universel et comparatiste. Néanmoins, il garde inaltéré le souvenir de son pays, accru par l'exil douloureux, qu'il interprète, dans la logique de sa mythologie personnelle, comme une " épreuve " initiatique.

Eliade nourrissait depuis la guerre le projet d'une monographie religieuse sur la Dacie, comme il note dans son " journal portugais ", le 12 janvier $1942^{7}$. Il ne présentera qu'en 1970 le recueil concernant son espace natal - De Zalmoxis à Gengis-Khan. Études comparatives sur les religions et le folklore de la Dacie et de l'Europe Orientale [dorénavant $Z G K$ ]. Dumézil saluera ainsi le livre : " [Eliade] se penche sur une patrie que trente années d'épreuves et de séparation lui ont peut-être rendue plus proche, plus intelligible. " Dans l'introduction, Eliade soulignait la pauvreté de la documentation ; pourtant, " nous avons essayé de décrypter dans les documents leurs significations profondes, maintes fois camouflées, dégradées ou oubliées 8 ". Car " nous disposons d'une tradition folklorique, et d'une tradition historique, importante aussi, mais dont les documents sont épars et vagues ; comment, à partir de cela, reconstruire les croyances des Daces 9 ?" .

livre engagé d'A. Laignel-Lavastine (Cioran, Eliade, Ionesco : l'oubli du fascisme. Trois intellectuels roumains dans la tourmente du siècle, Paris, 2002) a été farouchement attaqué en Roumanie, justement à cause de la démolition de l'icône d'Eliade. Voir, en général, la lucide biographie de F. TurCanu, Mircea Eliade. Le prisonnier de l'histoire, Paris, 2003. Lettre $\mathrm{n}^{\circ}$ XIV à Eliade, du 9 mars 1938 (éd. N. SPINETO, Mircea Eliade, Raffaele Pettazzoni. L'histoire des religions a-t-elle un sens? Correspondance 1926-1959, Paris, 1994, p. 117).

M. Eliade, Mémoires II. 1937-1960, Paris, 1988, p. 263-264 ; ID., " Lucian Blaga et le sens de la culture ”, in L'̂̀le d'Euthanasius, Paris, 2001 (= 1943), p. 171-177.

8 ELIADE, ZGK, p. 9-10.

9 M. EliADE, L'épreuve du labyrinthe. Entretiens avec C.-H. Rocquet, Paris, 1978, p. 117. 
Les deux premières études concernent la religion dace ; tout le reste regarde le folklore roumain. L'étude sur Zalmoxis est la première qui esquisse le destin de ce personnage dans l'histoire des idées. Cependant, son nationalisme (qu'il n'a jamais dépassé) ainsi que sa nostalgie sont évidents dans le recueil, surtout dans le traitement des deux légendes roumaines : Mioritza ("la petite brebis ») synthétise l'essence même de la "roumanité "; Mâ̂tre Manole, pourtant présente dans de nombreuses versions dans l'espace multiculturel des Balkans, serait, dans sa variante roumaine, "supérieure " et parfaite. Ces cultures rurales et archaïques conserveraient des traditions religieuses depuis le néolithique, à travers le « christianisme cosmique » à caractère anhistorique ${ }^{10}$. Plus tard, Eliade avouera qu'il s'agit « d'un livre très personnel en même temps qu'une expérience dans la méthode 11 ".

Dans la première étude, "Les Daces et les loups ", Eliade bâtit toute une théorie au sujet du rôle central que le loup aurait détenu dans la mythologie dace (une confrérie secrète de guerriers). Rien de cette théorie ne résiste à un examen plus approfondi 12 ; à la fin, les considérations sur "l'histoire mythique des Daces » disent beaucoup sur la vision et la conviction intimes d'Eliade, transmuées dans une philosophie de l'histoire appliquée à son propre pays.

La deuxième étude, "Zalmoxis » (p. 31-80), comporte deux dates, 1944 et 1969 ; la dernière marque une refonte totale de l'étude. Pour ce qui est de la bibliographie roumaine, il cite V. Pârvan (Getica, 1926, qui occupe une place de choix dans son rapport au passé dace), J. Coman, ainsi que les ouvrages récents, dans un évident désir d'actualiser la documentation. Pour Eliade, l'histoire rapportée par Hérodote est cohérente, bien qu'elle ait dénaturé la signification religieuse de ces faits. Une longue digression étale un scénario mythico-rituel éliadien : descendre aux Enfers comme une " mort initiatique ", "disparition " (occultation) et " réapparition " (épiphanie). Zalmoxis aurait instauré un culte eschatologique, centré sur l'immortalité de l'âme, dans une post-existence bienheureuse. Plusieurs digressions permettent à Eliade d'argumenter ses

10 F. TURCANu, "De l'histoire au mythe. La formation de l'image du Sud-Est européen dans l'œuvre de Mircea Eliade ”, Studia Politica 1 (2001), p. 185-192 ; "Il est troublant de constater que sous certains aspects la valorisation par Eliade du folklore et de son rapport avec les mythes et les symboles apparait comme l'accompagnement intellectuel de son engagement sans ambiguïté en faveur de la Garde de Fer roumaine» (p. 191).

11 Eliade, L'épreuve, p. 117. Dans l'analyse de Mioritza, très nationaliste, Eliade parle d'une " terreur de l'histoire " qui menace depuis toujours les Roumains, envers laquelle la réponse efficace serait la création spirituelle. Ce syntagme apparaît pour la première fois dans son “ journal portugais" (le 29 janv. 1944), avant de devenir célèbre dans Le mythe de l'éternel retour (cf. aussi TuRCANu, De l'histoire, p. 192). D'autre part, il écrivait en 1937 : "Ce n'est pas la matière illustrant une théorie qui en révèle la structure intime, mais la méthode, l'orientation spirituelle de l'auteur » [M. ELIADE, Cosmologie et alchimie babyloniennes, Paris, 1991, p. 12].

12 Eliade, $Z G K$, p. 13-30 [= Numen 6 (1959), p. 15-31]. 
thèses : les extatiques et les thaumaturges grecs ; les sacrifices humains. En accord avec certaines spéculations d'avant la deuxième guerre mondiale en Roumanie, il estime que le culte de Zalmoxis se prêtait à une christianisation presque totale ${ }^{13}$. Dans la dernière partie, Les métamorphoses de Zalmoxis (p. 75-80), il note la redécouverte des Daces comme " ancêtres mythiques ", avec Hasdeu, Pârvan, Blaga, ou même la "thracomanie ", redécouverte qui est " œuvre de culture et s'insère dans l'histoire des idées de la Roumanie moderne ». La fin de l'étude est donc élégante et très lucide. Selon l'historienne roumaine Zoe Petre : " [les] conclusions de Mircea Eliade et de ses collaborateurs, même si elles pèchent parfois par un excès de systématisation ou de comparatisme généralisé, [...] font preuve d'une vue équilibrée et d'une attitude critique envers les excès dilettantes de l'entre deux guerres, qu'Eliade dénonce fermement au tournant d'une phrase ${ }^{14}$. " Eliade donne pourtant l'impression qu'il croit vraiment à une importance spirituelle de Zalmoxis, dont le culte avait façonné la spiritualité roumaine. Malgré ses observations lucides quant aux dérives nationalistes des autres, lui non plus n’a pas échappé au mirage du dieu gète.

Cette vision sera résumée dans son Histoire des croyances et des idées religieuses (1978) 15. Dans le chapitre Les Thraces, "Grands Anonymes " de l'Histoire, dont le titre est un écho évident à Blaga (moins pour les lecteurs occidentaux, il est vrai), on apprend que « les Grecs avaient reconnu assez tôt l'originalité et la force de la religiosité thrace "; leur héritage religieux s'est conservé dans le folklore roumain et balkanique, par la "valorisation religieuse de la mort ". Le chapitre suivant, Zalmoxis et l'« immortalisation », est manifestement un abrégé de l'étude de 1970.

Eliade semble escamoter le problème du statut de Zalmoxis - réformateur ou dieu, même s'il semble opter pour la première variante. Certes, pour lui, il était plus important de démontrer que le culte était initiatique, suite à une révolution spirituelle (un autre concept qui lui est cher), donc, en dernière instance, conséquence de l'action d'une personnalité. La bibliographie utilisée par Eliade est abondante. Il reste cependant confus dans la présentation et l'interprétation des données, qu'il ne soumet pas vraiment à une critique historique. À l'exception d'Hérodote (dans la traduction de Legrand), il ne semble pas connaître les autres sources de première main. Il semble trop débiteur de ses devanciers, d'où il extrait les données anciennes, les références, parfois les commentaires mêmes. C'est ainsi qu'on observe des détails qui certifient cette connaissance relative des sources ; il lui arrive même de donner des références erronées.

13 Pourtant, EliAde ( $Z G K$, p. 74 n. 135) critique certains auteurs roumains (tel Coman), selon lesquels Zalmoxis avait anticipé, voire préparé, le christianisme. Z. Petre, "Le mythe de Zalmoxis", Analele Universității Bucureşti. Istorie 42-43 (19931994), p. 33. 175. 
Considérons le cas du théologien Jean G. Coman (1902-1987), selon lequel la religion gète aurait été un monothéisme " ethnique ». Sa position idéologique combinait le nationalisme avec une vision chrétienne-orthodoxe exacerbée. Plus tard, il affirma avoir suggéré à Eliade le nom de la revue Zalmoxis, où il avait publié un article sur Zalmoxis, largement utilisé par Eliade, et un autre sur Décénée, très idéologique ${ }^{16}$. À l'époque, l'helléniste D.M. Pippidi reprochait à Coman l'absence des règles les plus élémentaires de la méthode historique. Pippidi n'est pas étonné par le fait que ce théologien ait popularisé les mêmes opinions en roumain (dans la revue orthodoxiste Gândirea) et ensuite en français (dans Zalmoxis), mais " ce qui ne se comprend guère, c'est qu'il ait trouvé un directeur de revue disposé à les imprimer 17 ». Ce directeur n'était autre que Mircea Eliade ${ }^{18}$...

Une des sources théoriques inavouées est précisément le poète et le philosophe roumain Lucian Blaga (1895-1961), qui n'est cité que dans les chapitres concernant le folklore. Deux expressions sont péremptoires : (1) la " post-existence » rapportée à l'immortalité gète ; (2) les Thraces vus comme « les Grands Anonymes » de l'histoire. Or, le principe transcendant de la philosophie de Blaga était précisément le "Grand Anonyme ». Blaga discernait dans Mioritza la communion tragique et irréductible entre un territoire, le destin et le sacrifice prédestiné. Contrairement à la conception latiniste dominante, il a privilégié la " révolte de nos origines non-latines ». Sa valorisation de l'élément thrace (qui n'est pourtant pas thracomane, car il critique Coman) reléguait l'essence roumaine dans un monde anhistorique et atemporel, dans une existence organique, dans un long «boycott » de l'histoire, un monde où le christianisme était effacé devant les anciennes croyances païennes ${ }^{19}$. Toutes ces idées se retrouvent, sous des formes variées, chez Eliade.

Eliade annonçait dès l'introduction : "En effet, ce n'est que dans l'univers des valeurs spécifiques des chasseurs et des guerriers, notamment à la lumière des rites initiatiques, que le nom ethnique des Daces révèle sa signification religieuse primitive.

16 Cf. M. Handoca, Convorbiri cu şi despre Mircea Eliade, Bucarest, 1998, p. 136 ; J. Coman, Zalmoxis; ID., "Décénée", Zalmoxis 3 (1940-1942), p. 103-160. D.M. PipPidi, c.r. de J. Coman ("Zalmoxis ”), Revista Clasică 15 (1943), p. 117-118 ; ID., c.r. de J. Coman ("Décénée ”), Revue Historique du Sud-Est Européen 23 (1946), p. 340-342.

Lequel écrivait à C. Noica, le 26 avril 1942 : "Ce volume de Zalmoxis [n 3], je voudrais qu'il soit le plus roumain possible : folklore, ethnographie, préhistoire. Dis à Coman de préparer quelque chose de dace [...] Dis-leur qu'il s'agit d'une action roumaine, en premier lieu. " [M. Eliade, Europa, Asia, America... Corespondență, II, Bucarest, 2004, p. 357]. Pourtant, Eliade connaissait très bien Coman, car il écrivait au même Noica le 22 février 1943 : "Comment se présente Deceneu de Coman ?! L'as-tu prié de ne pas écrire plus de 25 pages? Si c'est le texte de Gândirea, il sera donc regrettable. " (ELIADE, Jurnalul, II, p. 475-476).

19 C. Karnooh, Linvention du peuple. Chroniques de Roumanie. Essai, Paris, 1990, p. 200221 ; K. Hitchins, Rumania. 1866-1947, Oxford, 1994, p. 305-314. 
De même, le culte de Zalmoxis ne devient compréhensible qu'après qu'on a dégagé le sens initiatique de l'occultation et de l'épiphanie du dieu ${ }^{20}$. "On n'est point étonné qu'il favorise exactement les thèmes récurrents de son ouvre 21 dans l'étude sur Zalmoxis : initiations, sociétés secrètes (Männerbünde), culte aristocratique, sacrifice humain comme "mort créatrice ", extase, pratiques ascétiques.

Or, la thèse d'Eliade repose largement et davantage sur ses propres convictions et suppositions que sur des démonstrations. Si le récit de la " catabase » est la version des Grecs du Pont, et s'il est, en outre, intégré dans une grille pythagoricienne (Eliade en est bien conscient), pourquoi alors le considérer comme un témoignage sur les croyances et les pratiques rituelles gètes? Pourquoi mettre en rapport le "mythe " (catabase, occultation, épiphanie) avec le « rite » (sacrifice du messager) 22 ? Qui plus est, sa vision sur les " mystères " était traditionnelle, chargée de toutes ses préférences personnelles. Elle n'est plus acceptable de nos jours; dans les mystères il n'y avait pas de foi dogmatique en un triomphe sur la mort, ni une dévalorisation de la vie non plus 23 .

La thèse initiatique d'Eliade, alors au sommet de son prestige, devient rapidement une opinion commune ${ }^{24}$. Zalmoxis est désormais vu comme un réformateur religieux, instituant un culte mystérique, aristocratique et initiatique, de facture "supérieure ". Dans l'historiographie occidentale, elle ne semble pas avoir été soumise à une vérification quelconque. Et l'origine roumaine d'Eliade n'a rien fait d'autre que d'apporter un argument supplémentaire.

Comme le montre Dubuisson, l'œuvre abondante d'Eliade a exercé et continue encore d'exercer une influence multiforme et diffuse. Il lui reproche, à juste titre, des graves défauts de méthode : choix arbitraires et simplificateurs, généralisations abusives, interprétations contestables. La critique la plus forte d'Eliade, et pleinement justifiée, vise son mépris foncier et délibéré du conditionnement historique ${ }^{25}$. Force est de constater que presque toutes ces critiques valent aussi pour $Z G K$.

Il est utile de regarder de plus près l'intérêt d'Eliade pour l'héritage de ses ancêtres assumés. En 1937, il cherche à se justifier auprès des journalistes nationalistes l'accusant de ne pas avoir abordé le fameux «spécifiquement national », le " roumanisme ".

ELIADE, ZGK, p. 10.

D. Dubuisson, Mythologies du XXe siècle (Dumézil, Lévi-Strauss, Eliade), Lille, 1993, p. 281-288.

Cf. aussi HarTOG, Miroir, p. 190.

W. BURKERT, Les cultes à mystères dans l'antiquité, Paris, $2003^{2}$ (= Harvard, 1987).

K. W. TRePtow, "A Study in Geto-Dacian Religion : The Cult of Zalmoxis ", in From Zalmoxis to Jan Palach. Studies in East European History, New York, 1992, p. 5-22 ; J. VIDAL, s.v. Zalmoxis, in P. POUPARD (éd.), Dictionnaire des religions, II, Paris, 19933, p. 2151-2153 ; Y. Ustinova, “'Either a Daimon, or a Hero, or Perhaps a God' : Mythical Residents of Subterranean Chambers ", Kernos 15 (2002), p. 267-288 ; J. BREMMER, s.v. Zalmoxis, DNP, XII2, 2002, col. 691.

Dubuisson, Mythologies, p. 18 ; 215-303 ; BorgEaud, Exercices, p. 181. 
Loin de critiquer une telle attitude, Eliade met en évidence la problématique de l'autochtonie. "Le peuple roumain, qui n'a eu ni un Moyen Âge glorieux (dans le sens occidental) ni une Renaissance et qui n'a donc pas été de ceux qui on "fait" l'histoire et la culture européennes, a une préhistoire et une protohistoire égales en valeurs à celles de n'importe quelle nation européenne importante, et un folklore incontestablement supérieur à toutes les autres. Aujourd'hui, la science roumaine a l'occasion unique de mettre en valeur la spiritualité et l'histoire secrète de notre nation ${ }^{26}$. "Tel était son programme idéologique en 1937, et, à la lecture de $Z G K$, force est d'observer qu'Eliade est nourri par le même esprit. Dans l'introduction, il écrivait : "Car le culte de Zalmoxis, par exemple, aussi bien que les mythes, les symboles et les rituels qui informent le folklore religieux des Roumains, plongent leurs racines dans un monde de valeurs spirituelles qui précède l'apparition des grandes civilisations du Proche Orient antique et de la Méditerranée ${ }^{27}$."

Pour l'ensemble de son œuvre publiée en Occident, Eliade va cependant reformuler certaines de ses positions antérieures, dans une version transcendant la perspective nationaliste pour se réclamer d'un humanisme universel, à travers la théorie de l'archaïque et de l'archétype ${ }^{28}$. Or, dans $Z G K$, bien qu'il soit moins nationaliste qu'autour de 1940, Eliade observe une position beaucoup plus conservatrice que dans le reste de son œuvre, vision marquée par l'exil : le culte de Zalmoxis préfigure donc l'unicité et la profondeur spirituelle qui semblent prédestinées au peuple roumain.

\section{La Garde de Fer : glorification du sacrifice et de la mort}

Dans ZGK, Eliade rappelle les propos d'un de ses amis de jeunesse, le poète Dan Botta, sur la "nostalgie de la mort » et sur le thracisme ethnique des Roumains, thème développé dans Ondoiement et mort (1936), dédié à Eliade ${ }^{29}$. On y rencontre Zalmoxis, dieu pur et parfait, adoré par les Thraces monothéistes, et préfigurant le christianisme. Chez les Thraces, l'idée de la mort était liée de l'idée du bonheur absolu, similaire aux noces cosmiques chantées par la Mioritza et à la fascination de la mort propre à la légende du Maître Manole. On ne sera point surpris de retrouver le même schéma interprétatif chez Eliade.

Cette fascination pour la mort chantée par Botta n'est pas singulière à l'époque, bien au contraire, ni l'idée du mariage avec la mort, choisie par de nombreux jeunes engagés dans l'un des mouvements d'extrême droite les plus durs de l'Europe. Le

ELIADE, ZGK, p. 9.

BORGEAUD, Exercices, p. 185 ; 191-196.

ELIADE, ZGK, p. 225-226 ; ID., "Fragment pentru Dan Botta ”, in Prodromos 7 (1967), p. $19-21$ = D. BOTTA, Unduire şi moarte, Jassy, 1995, p. 9-14. 
modèle intellectuel de la jeunesse était le professeur charismatique Nae Ionescu 30 (1890-1940), maître (trop) vénéré d'Eliade, et qui a charmé même un esprit aussi lucide que celui de l'écrivain juif M. Sebastian.

Dans les années 1930, on assiste à un premier processus de rhinocérisation : il touche la partie la plus visible des intellectuels roumains ${ }^{31}$, qui ont graduellement adhéré, pour diverses raisons, à l'idéologie de la tristement célèbre Légion de l'Archange Michel ou la Garde de Fer. Parmi eux, plusieurs écrivains qui s'affirmeront à l'étranger, comme V. Horia, E. Cioran et M. Eliade. Si en Allemagne le nazisme était anti-chrétien, voire néo-païen, ou si en Italie le fascisme vantait la grandeur romaine, en Roumanie la mystique légionnaire était ultra-religieuse, plus précisément orthodoxe (afin de se démarquer davantage encore de l'Occident catholique, protestant ou athée). D'autre part, les années 1930 marquent une période de "spiritualisation " graduelle du spectre intellectuel roumain ; la quête de l'originaire et de l'autochtone aboutit parfois à un dacisme manifeste et à un autochtonisme démesuré. Cioran raconte une entrevue de 1934 avec C.Z. Codreanu, le chef charismatique de la Légion, lors de laquelle le "Capitaine » lui a " exposé ses vues sur la manière de faire revivre les vertus daces 32 ".

Il fallait donc dé-paganiser (ou christianiser) la religion gète : Zalmoxis devient le réformateur d'une religion monothéiste, ascétique, dominée par des élites, dans une unité organique avec le peuple, religion qui promettait l'immortalité et grâce à laquelle les ancêtres ne redoutaient pas la mort. Vanter l'esprit de sacrifice des Daces et leur " mort pour la Patrie " préfigurait ainsi la vulgate légionnaire : ses membres, Chrétiens et Roumains fanatiques, aiment la mort et vont à sa rencontre, contre les ennemis de la Nation et de Dieu (capitalistes, démocrates, Juifs, bolchéviques, franc-maçons ou athées). La mystique de la mort et du martyre sont les convictions les plus affichées des légionnaires, malheureusement transposées dans la réalité.

Dans ce cadre de pensée, les affirmations d'un chef légionnaire, Al. Cantacuzino, sont plus que transparentes : «Rien ne détermine, ne définit ou ne différencie le plus exactement une vie, rien ne façonne plus manifestement un être humain ou la société que la manière de voir et de recevoir la mort. Une nation d'hommes indifférents à la mort est vouée à des victoires illimitées... Dans le monde légionnaire, nous nous vantons non seulement du mépris de la mort, mais nous nous glorifions de l'amour de la mort. La conception légionnaire de la mort fraternise, vingt siècles après, avec les

30 M. Eliade, “Nae Ionescu 1890-1940 (treizeci de ani de la moarte) ”, in Prodromos 10 (1970), p. 19.

31 Cf. E. IONESCO, in L'Express, $\mathrm{n}^{\circ}$ 1004, 11 oct. 1970 : « Le rhinocéros, c'est l'homme des idées reçues... Je l'avais vécu, une première fois, en Roumanie, lorsque l'intelligentsia devenait peu à peu nazie, antisémite, "Garde de Fer "... Les professeurs de faculté, les étudiants, les intellectuels." 
enseignements de Zamolxis qui a prêché parmi les Géto-Thraces le culte de l'immortalité de l'âme 33 ..."

La religion des Daces et l'image de Zalmoxis connaissent donc, dans les années trente, un processus de spiritualisation à des fins idéologiques : préfigurations du "spécifiquement national ", modèles du passé, proposés pour un futur d'exception destiné au peuple roumain. Certes, dans la monographie d'Eliade, ce radicalisme des années 1930 est atténué, mais, pour parler avec son auteur, l'essence en est la même : les Daces sont un peuple méprisant ou aimant la mort, adorant un personnage exceptionnel, qui leur promet l'immortalité, dans un culte très spirituel. Cette vision éliadienne n'est pas "légionnaire ", et il faut se garder de coller trop facilement des étiquettes, mais elle partage, à n'en pas douter, le même nationalisme culturel qui a connu une diversité d'expressions dans la Roumanie d'entre-deux guerres.

\section{Métamorphoses d'Eliade : la guerre, l'exil et la récupération culturelle}

Comme ses exégètes ne cessent de l'observer, la période roumaine est essentielle dans la formation intellectuelle d'Eliade. Le jeune Eliade notait avec satisfaction le reflux du positivisme et la " passion des élites actuelles pour la préhistoire, les races, les religions, les mythologies, les symboles 34 ». "Nous participons à un peuple originaire, dont la gloire précède la gloire de Rome... ». Et Eliade d'exprimer à la fois un constat et un vœu : "Aujourd'hui [1937], la fascination des Daces dépasse l'intérêt scientifique ; tout nous pousse à croire que la soif d'"originaire" et de "local" va s'approfondir dans la spiritualité roumaine ${ }^{35}$."

Nommé attaché culturel à Lisbonne, Eliade vise à renforcer le lien entre les deux pays latins. En 1942, il publie en Roumanie un ouvrage sur Salazar ; et, l'année suivante, une brève histoire des Roumains, œuvre à la fois de propagande et de vulgarisation, en espagnol et en portugais ${ }^{36}$. Sa présentation de l'histoire roumaine est très nationaliste, fidèle à l'esprit de son époque ; mais elle témoigne largement de l'empreinte personnelle d'Eliade, par les concepts-clé qui lui sont chers : spiritualité, culture, religion, et surtout le destin et la mission du peuple roumain. La naissance du peuple roumain est placée

33 Al. CANTACUZINO, Intre lumea legionară şi lumea comunistă, Bucarest, 1935, p. 14. Après avoir combattu en Espagne comme volontaire dans les rangs franquistes, Cantacuzino est exécuté le 22 septembre 1939, dans un effort désespéré des autorités roumaines d'éliminer physiquement les chefs du Mouvement Légionnaire, après l'assassinat du premier ministre Armand Calinescu par des militants gardistes, le 21 septembre. $(=1939)$. 
Sous le signe de Zalmoxis; des années plus tard, dans ZGK, il la mettra Sous le signe du Loup, c'est-à-dire un peuple prédestiné aux invasions, aux exils et aux guerres (dans sa vision de l'Histoire marquée par l'exil et l'avènement du communisme en Roumanie). Le peuple roumain, oasis de latinité rattachée à l'Occident, a une mission historique, en tant que peuple de frontière, défenseur de la chrétienté et de l'Europe : il mène la guerre sainte, qui a une "signification spirituelle ", contre les Soviets et leur " mysticisme asiatique ». Eliade insiste sur deux mythes centraux de la spiritualité roumaine (Maître Manole et Mioritza), qui seront développés dans $Z G K$.

Eliade appartient au groupe d'exilés roumains intéressés par leur passé : partis d'une même image idyllique esquissée par Pârvan, ils arrivent à recomposer, chacun dans son domaine ${ }^{37}$, une image encore plus mythique de la Dacie, d'une haute spiritualité. L'éloignement douloureux de leur patrie ne faisait qu'éloigner encore plus, dans des profondeurs mystérieuses, cette Dacie où se cachait Zalmoxis.

La fortune d'Eliade commence à toucher aussi la Roumanie. Dès le début des années 1970, on assiste au retour en force d'une idéologie autarciste et autochtoniste sous le long règne de Ceauşescu. Dans cette quête d'une nouvelle légitimité s'inscrit aussi la réhabilitation et la récupération d'Eliade par la « mise en valeur critique de l'héritage culturel 38 ". Les idées de $Z G K$ circulaient dès sa parution française, mais le livre ne sera traduit en roumain que dix ans après (1980) ${ }^{39}$ : on était donc « libre » de suivre Eliade. L'étude d'Eliade ne faisait rien d'autre que renforcer et légitimer une fois de plus la fascination exercée par Zalmoxis, par son double prestige, de compatriote et de savant occidental reconnu.

37 Par exemple deux de ses amis : (1) A. Busuioceanu (1896-1961), exilé en Espagne : Zamolxis sau mitul dacic în istoria şi legendele spaniole, Bucarest, 1985 ; (2) V. HorIA (1915-1992), qui publie en 1960 Dieu est né en exil. Journal d'Ovide à Tomes, roman couronné du prix Goncourt. Le prix ne sera jamais décerné à cause du passé politique de son auteur (ancien gardiste). On y rencontre Zalmoxis comme dieu unique d'une religion dace préfigurant le christianisme et fascinant l'exilé Ovide.

K. VERDERY, National Ideology Under Socialism. Identity and Cultural Politics in Ceausescu's Romania, Berkeley-Los Angeles-Oxford, 1991, p. 125 ; Laignel-LaVAStine, Cioran, p. 465-470 ; TurCANu, Eliade, p. 468-471 ; F. TurCANU, " Mircea Eliade et la renationalisation de la mémoire collective dans la Roumanie de Ceausescu ", in B. JEWSIEWICKI (éd.), Travail de mémoire et d'oubli dans les sociétés postcommunistes, Bucarest, 2006, p. 46-63.

Lettre à M. HANDOCA (11 sept. 1978), où Eliade fait une allusion cachée à Ceauşescu : "Je regrette que Zalmoxis soit à nouveau différé (serait-ce à cause de GengisKhan ?) " [" La réception de l'œuvre de Mircea Eliade en Roumanie ”, in J. RIESN. SPINETO (éd.), Deux explorateurs, p. 293]. 


\section{I.P. Culianu : Eliade, le nouveau Zalmoxis, et ses occultations}

Examinons le cas d'un autre exilé, Ioan Petru Culianu (1950-1991). Fasciné par le modèle d'Eliade, il part en Italie (où Ugo Bianchi l'oriente vers l'étude des dualismes), pour occuper enfin la chaire d'Eliade à Chicago, où il sera assassiné en mai 199140 . Parmi d'autres savants roumains sollicités pour la Encyclopedia of Religion (ER), Eliade réserve à Culianu et à $\mathrm{C}$. Poghirc (autre exilé) les entrées sur la religion des Thraces et des Daces ; leur vision est encore plus spiritualiste ${ }^{41}$.

Ce n'est que récemment qu'une documentation de plus en plus riche nous restitue la relation fort complexe entre Eliade et Culianu. Ce dernier prépare très tôt une biographie d'Eliade (1978, en italien). L'on croyait que ce n'était que beaucoup plus tard qu'il connaîtrait la vérité sur le passé légionnaire d'Eliade. Or, dans son journal inédit, Culianu notait, le 10 janvier 1978 : " Je ne peux pas être solidaire avec lui [Eliade], l'idéologie légionnaire m'est aussi étrangère que l'idéologie communiste. Elles se ressemblent considérablement [...] La surprise que Mircea Eliade ait été le partisan d'un mouvement totalitaire et qu'il soit resté toute sa vie fidèle à sa mythologie me comble d'amertume ${ }^{42}$. " Plus que la découverte du passé légionnaire d'Eliade, ce sont l'attitude et les réponses (1 ${ }^{\text {er }}$ mars 1978) du maitre révéré qui attristent le plus le jeune Culianu : "Je n'ai pas répondu, et ne répondrai jamais ${ }^{43}$."

Culianu s'y conforme : dans la monographie Eliade s'en sort bien ; il est présenté comme un démocrate de gauche 44 ! Notons un autre détail : Eliade écrivait à Culianu, au sujet de ses articles dans la presse de l'exil : "Même si j'étais, comme tous les autres, "engagé", et que j'attaquais le régime du pays - ma thèse était la suivante : les exilés ne peuvent rien faire immédiatement sur le plan politique; notre seule "arme politique" est la culture ; toute création roumaine importante constitue une chance pour la "survie de ma nation". " C'est ici, condensée, la fameuse thèse de la résistance par la culture, chérie aussi bien par les exilés que par l'intelligentsia roumaine du pays ${ }^{45}$. Or, par rapport à l'implication massive des intellectuels des pays d'Europe Centrale dans la dissidence

40 Crime encore non résolu : les soupçons portent sur la Garde de Fer en exil, sur l'ancienne Securitate, ou même une combinaison des deux. Voir T. ANTON, Eros, Magic and the Murder of Professor Culianu, Chicago, 1996.

I. P. Culianu-C. PoghirC, s.vv. Geto-Dacian Religion, ER, V, 1987, p. 537-540; Thracian Religion, ER, XIV, p. 494-497 ; Zalmoxis, ER, XV, p. 551-554 ; M. Eliade-I.P. CoulianO, Dictionnaire des religions, Paris, $1997^{2}$ (= 1990), p. 291-294 (Religion des Thraces, rédigé uniquement par Culianu).

I. P. Culianu, Dialoguri întrerupte. Corespondență Mircea Eliade-Ioan Petru Culianu, Jassy, 2004, p. 127.

Ibid., p. 134 (lettre $\mathrm{n}^{\circ} 42$ ).

Ce qu'Eliade n'a certainement jamais été ; dans les années 1930, les intellectuels de gauche étaient des rarae aves en Roumanie.

Culianu, Dialoguri, p. 131-132 (lettre n 41, du 13 févr. 1978). 
anticommuniste, l'intelligentsia roumaine a choisi cette " résistance culturelle ", qui n'était dans la plupart des cas qu'un retour à un nationalisme culturel. Dans les années 1980, l'intelligentsia, qui avait pratiqué en effet le jeu du pouvoir, changeant le marxisme de circonstance en un nationalisme patent, constate la faillite de tout espoir 46 .

\section{Le « dossier » Eliade en Roumanie}

Avec le "dossier " Eliade en Roumanie, on aborde un domaine très sensible. De son vivant, le succès occidental d'Eliade lui a valu en Roumanie une admiration sans bornes. Courtisé par le régime de Ceauşescu, célébré et censuré en même temps ${ }^{47}$, Eliade restera toujours, pour les intellectuels de Roumanie, une figure lointaine et d'autant plus fascinante, et une alternative - bientôt la seule -, à la fois roumaine et occidentale pour penser la culture. Après 1989, son culte plongea toute une génération dans une admiration béate, déterminant une vogue pour l'histoire des religions. En même temps, son passé politique était fermement ou élégamment dissimulé ${ }^{48}$; car la génération des années 1920-1930 devint le modèle de la jeunesse cultivée. L’exil et son anticommunisme, mais davantage sa réputation de plus grand historien des religions au monde, l'ont placé sur un socle mythique, en tant que nouvel évangile par lequel jure l'intelligentsia roumaine. Ce n'est qu'assez récemment que des voix plus critiques osent discuter en Roumanie son passé politique, mais sans jamais mettre en doute la validité de sa production scientifique 49 .

Or, la lecture que je propose de ses écrits de Roumanie et d'Occident au sujet de la religion dace (et du folklore roumain) montre combien l'idéologie spiritualiste dans laquelle Eliade baignait, avec toute une génération, dans les années 1930, ne l'a point quitté. Elle est perceptible dans tous ses écrits sur des sujets roumains, le tout imprégné d'érudition, au sein d'un système herméneutique aspirant à une valeur universelle.

46 KaRNOOH, L'invention, p. 182-191 ; A. LAIGNEL-LAVASTINE, “ Le national-communisme en Roumanie ”, in D. CEFAÏ (éd.), Cultures politiques, Paris, 2001, p. 341-364.

47 Lettre du 10 mars 1974 à H. Stamatu (poète légionnaire) : "Dans la dernière année, s'est déchaînée une sorte d'admiration générale et délirante de mon activité (et personne) en Roumanie " [ELIADE, Europa, III, 2004, p. 165].

TurCanu, Eliade, p. 1-2.

49 D'une défense totale d'Eliade, on est passé à la thèse de la non-contamination de son œuvre scientifique. Son engagement légionnaire est reconnu, mais pardonné utilisant la justification donnée par Eliade lui-même : il aurait été séduit par l'aspect quasi-religieux de la Garde de Fer, car « toute révolution est spirituelle ». Le livre de Laignel-Lavastine ou bien la critique de Dubuisson sont rejetés avant d'être interrogés de plus près, car ils sont « détracteurs » d'Eliade. Cela s'explique par le modèle volontairement élitiste de l'intelligentsia roumaine actuelle. 
Dans une lettre envoyée en 1943 au critique littéraire T. Vianu, Eugène Ionesco constatait : " J'ai l'impression, en relisant Blaga, Eliade, etc., qu'il en ira toujours ainsi chez nous [les Roumains] : une volonté farouche de se séparer de l'universel, une hostilité irréductible à l'égard de l'Occident, une nouvelle mythologie balkanique. Rien d'autre. Une province. Incapable d'adhérer aux grandes unités. L'influence de la culture française s'est avérée si superficielle 50 !"

Très pertinente pour l'opinion actuelle en Roumanie sur le "problème Eliade " me semble une conférence de l'ethnologue Andrei Oişteanu, intitulée Mircea Eliade, entre orthodoxisme et zalmoxisme (New Europe College de Bucarest, 3 juin 2002) 51. Elle n'est qu'une habile apologie, qui montre comment même les intellectuels roumains les plus ouverts et occidentalisés ont du mal à assumer et expliquer le passé d'Eliade, et encore moins à toucher à son mythe. Plus grave encore, même s'ils commencent à accepter l'épisode légionnaire d'Eliade - jugé " passager »-, ils n'acceptent aucune influence sur son " œuvre scientifique ", qui reste intangible. Oişteanu part du constat que le fascisme roumain est une exaltation du christianisme orthodoxe. Il estime dès le début qu'Eliade n'est pas victime de la thracomanie (ce qui est vrai), car ses deux études sur les Daces et sur Zalmoxis sont des " recherches exemplaires d'histoire des religions et des mentalités ». Selon Oişteanu, c'est seulement Coman qui a idéalisé les Gètes. Enfin, seule la pièce de théâtre Iphigénie (décembre 1939, représentée en février 1941) comporte des connotations politiques. S'il critique avec raison la lecture en clé antisémite faite par Dubuisson, Oişteanu estime en revanche qu'Eliade n'aurait pas fait de compromis politiques dans la partie la plus importante de son œuvre, l'histoire des religions. De ce fait, proposant la fausse dichotomie orthodoxisme/œuvre scientifique, il peut convenablement ranger Eliade dans cette dernière. Cependant, le problème est plus complexe. Il me semble évident qu'Eliade partageait, de même que presque tous ses contemporains, la fascination pour la spiritualité dace qui commence avec l'idéalisme de Pârvan, le stylisme de Blaga ou le spiritualisme affiché de Coman.

Eliade a beaucoup aimé décrire Zalmoxis comme un dieu maître des occultations. Culianu a même présenté son modèle comme le nouveau Zalmoxis des Roumains : «Pour les Roumains, Mircea Eliade se confond avec Zalmoxis : il a disparu de leur horizon et pourtant il leur donne des signes ${ }^{52}$. " Eliade parlait des occultations de Zalmoxis (voir le titre de la version anglaise, Zalmoxis, the Vanishing God), lui qui ne faisait rien d'autre que d'occulter son passé. Cette occultation consciente, voire délibérée, va hanter dorénavant la postérité scientifique de Mircea Eliade ; elle apporte déjà un nouveau "boycott de l'histoire ", non pas contre un peuple roumain toujours victime

50 Lettre du 23 déc. 1943, cf. Laignel-LaVASTine, Cioran, p. 360-361.

51 A. Oişteanu, " Mircea Eliade between Political Journalism and Scholarly Work ", Archaus 8 (2004), p. 323-340.

52 I.P. Culianu, Mircea Eliade, Bucarest, 1995, p. 259. 
d'injustices, mais contre un savant qui n'a pas su assumer son passé. Ou, selon le jugement général de C. Karnooh (1990) : " À vouloir toujours habilement "résister à la terreur de l'histoire", à inventer des "méthodes de camouflage", à s'attribuer le rôle confortable de l'éternelle victime des puissants, l'intelligentsia roumaine a perdu tout sens d'appartenance et de responsabilité dans le mouvement même de l'histoire ${ }^{53}$. "

DAN DANA

30, rue du Plateau

75019 Paris

ddana_ddan@yahoo.com

53 KaRNOOH, Linvention, p. 189. 